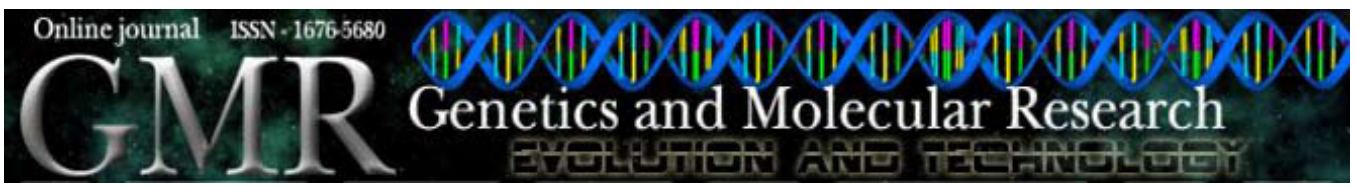

Link to retraction noticed

\title{
Genetic and biochemical analyses of sensor kinase A in Bacillus subtilis sporulation
}

\author{
J.C. Nguele ${ }^{1,2}$, P. Eswaramoorthy ${ }^{1}$, M. Bhattacharya ${ }^{1}$, E. Ngou-Milama ${ }^{2}$ \\ and M. Fujita ${ }^{1}$
}

${ }^{1}$ Department of Biochemistry, University of Houston, Houston, TX, USA

${ }^{2}$ Département de Chimie-Biochimie, Biologie Cellulaire \& Moléculaire,

Faculté de Médecine, Université des Sciences de la Santé, Libreville, Gabon

Corresponding author: J.C. Nguele

E-mail: jcnguele@yahoo.com

Genet. Mol. Res. 9 (1): 573-590 (2010)

Received December 7, 2009

Accepted January 15, 2010

Published March 30, 2010

ABSTRACT, Temporal and spatial regulation of gene expression during endospore formation in Bacillus subtilis prompted us to investigate the molecular mechanisms that coordinate the phosphorelay. We targeted KinA for random mutagenesis. In addition, we constructed KinA-GFP transcriptional fusions for verification, via fluorescence. Four distinct types of sporulation-defective mutants were identified as inactive (no sporulation), hypoactive (low sporulation efficiency), isoactive (same efficiency as wild-type), and hyperactive (high efficiency) mutants. Surprisingly, the $\beta$-galactosidase activity of hyperactive mutants was barely greater than that of the wild-type strain; the only noticeable difference was early synthesis of KinA, which could allow them to activate Spo0A precociously, undergo sporulation earlier, and yield more spores. There was no fluorescence emission by the spore-defective mutant, which confirmed the presence of a truncated KinA (nonsense mutation) in inactive strains; other mutants harbored missense or silent mutations. We determined the nucleotide sequences of KinA mutants and found a conserved C-terminus region; more variability was observed in the $\mathrm{N}$-terminus region, involving the PAS-A and PAS-C domains. We speculate that PAS-A, notwithstanding its ATPase activity, has only a minor role in KinA activity, whereas PAS-B was found to be indispensable. Our results emphasize the importance of 
temporal coordination of gene expression during the sporulation process and corroborate the necessity of Spo0A phosphorylation by KinA, which stimulates SpoIIG expression. We further propose a novel hypothetical model that purposely dubbed the "C-shaped intertwined model", which requires both homodimerization and spatial proximity between PAS-A and histidine $\mathrm{H}_{405}$ of two different KinA molecules.

Key words: KinA; Sporulation; C-shaped intertwined model; Bacillus subtilis

\section{INTRODUCTION}

Sporulation in Bacillus subtilis is principally governed by spo0A, which encodes for a member of the response regulator family of transcription factors, which upon reaching a threshold level of phosphorylation triggers the transcription of genes active at the onset of sporulation (Molle et al., 2003a; Fujita and Losick, 2003; Fujita et al., 2005). Over 125 genes are involved in this process, the transcription of which is temporally and spatially controlled by four DNAbinding proteins and five RNA polymerase sigma factors (Stragier and Losick, 1996). Several physiological factors figure prominently in this intricate system. The main stimulus for sporulation is known to be starvation (Piggot and Losick, 2002). In nature, this process is initiated under nutrient depletion (Dawes and Mandelstam, 1970; Piggot and Hilbert, 2004; Fujita et al., 2005). It is also important that the population densities be high (Grossman and Losick, 1988).

However, no single nutritional effect acts as the trigger. Rather, the cell has an extremely complex and sophisticated decision-making apparatus, which monitors a huge range of internal and external signals. Most response regulators are directly phosphorylated by KinA, the principal cognate sensor-kinase that carries out autophosphorylation on a histidine residue and then transfers the phosphoryl group to the aspartyl residue in the response regulator. It is principally governed by a sequential series of phosphorylation reactions, termed "phosphorelay", catalyzed by KinA and major downstream regulators, Spo0F, Spo0B, and Spo0A. Components of this essential cell cycle regulatory network are also found amongst medically important microorganisms, including Clostridium, Bacillus cereus, and B. anthracis (Burbulys et al., 1991).

Spo0A synthesis is controlled transcriptionally, and the activity of the resulting protein is regulated by phosphorylation. Transfer of phosphate to Spo0A is regulated by a complex network of interactions. Several kinases (KinA, KinB, KinC, KinD, and KinE) probably each respond to a different stimulus (Fabret et al., 1999). Phosphorylated Spo0A (hereafter referred to as $\mathrm{Spo} 0 \mathrm{~A} \sim \mathrm{P}$ ) is an essential regulator of sporulation, and it works by activating the transcription of several key sporulation-specific genes (Grossman, 1995; Molle et al., 2003b). Recent data have shown that Spo0A is probably also an important factor in sporulation during early mothercell development as a mother cell-specific transcription factor (Fujita and Losick, 2003).

The other key regulator of sporulation is an alternative sigma factor, $\sigma^{\mathrm{H}}$, which interacts with the RNA polymerase core enzyme and directs it to initiate transcription in the pre-divisional cell at an early stage of sporulation (Weir et al., 1991). Spo0A P is known to stimulate the production of $\sigma^{\mathrm{H}}$ through an indirect pathway involving the repressor $\mathrm{AbrB}$, which, in turn, directly stimulates the transcription of the gene for spoOA and kin $A$. In addition, both Spo0A P and $\sigma^{\mathrm{H}}$ stimulate the transcription of the gene for the phosphotransferase protein SpoOF (Predich et al., 
1992). Such a self-reinforcing cycle would lead to both preferential synthesis of Spo0A and phosphorylation of the response regulator in the sporulating cell. Other studies indicate that a certain threshold concentration of Spo0A P is necessary to initiate sporulation and that the activity of the phosphorelay determines the threshold level for autostimulation of Spo0A (Burbulys et al., 1991; Fujita and Losick, 2005). Activation of $\sigma^{\mathrm{H}}$ and Spo0A in the pre-divisional cell leads to expression of factors important for axial filament formation, asymmetric division, and compartmentalization of gene expression (Levin and Losick, 1996; Ben-Yehuda et al., 2003). The phosphorelay is also subject to negative regulation. In order to ensure that sporulation occurs only under the appropriate conditions, the phosphorelay must integrate different intracellular and extracellular signals. The mechanisms responsible for this integration are regulated by cell density and nutrient starvation. Efficient sporulation requires high cell density (Grossman and Losick, 1988).

When cell density is low, the Rap (response regulator aspartyl phosphatase) proteins RapA, RapB (Perego et al., 1994), and RapE (Jiang et al., 2000), which are efficient phosphatases, dephosphorylate $\mathrm{Spo} 0 \mathrm{~F} \sim \mathrm{P}$, preventing Spo0A activation. As cell density increases, the processed peptides are imported by oligopeptide permease and inhibit the activity of RapA and RapE. Thus, inhibition of the Rap proteins prevents dephosphorylation of SpoOF $\mathrm{P}$ and allows phosphorylation of Spo0A and the initiation of sporulation when cell density is high (Jiang et al., 2000).

In addition to high cell density, a dramatic drop in the concentration of GTP and GDP correlates with the onset of sporulation, and inhibition of GMP synthesis by decoyinine treatment induces sporulation in the absence of nutrient starvation (Mitani et al., 1977). CodY has been identified as the key sensor of guanine nucleotide levels. Disruption of the $\operatorname{cod} Y$ gene allows sporulation to occur in the presence of excess nutrients, and the ability of the CodY repressor to bind DNA correlates with the GTP concentration. As a consequence, when GTP levels drop upon entry into stationary phase, CodY-regulated genes, such as phrA, phrE, and kinB are derepressed. These genes are all positive regulators of the phosphorelay (Ratnayake-Lecamwasam et al., 2001).

Within a sporulating culture of $B$. subtilis, some cells initiate this developmental program, while other cells do not. Therefore, initiation of sporulation appears to be a regulatory process with a bistable outcome (Veening et al., 2005). However, it turns out that when populations of sporulating cells are examined, the activation of Spo0A appears to be quite graded: a low concentration of Spo0A P triggers early genes in the regulon, and a higher concentration produces a late-gene response (Fujita et al., 2005; Fujita and Losick, 2005). In this respect, the behavior of the sporulation system resembles that of thermodynamic systems: the discontinuous transition from growing to sporulating-state is similar to a phase transition of water, with chemical signaling instead of thermal shift driving transitions between two states. The general concept of feedback regulation is that signaling pathways typically convert a graded, analogue signal into a binary cellular output (Becskei et al., 2001; Ferrell Jr., 2002; Hasty et al., 2002; Isaacs et al., 2003). Notwithstanding the prolific findings on sporulation in all previous studies, none had successfully uncovered at the molecular level the early steps of the phenomenon or the nature of the putative triggering signals.

In this study, we investigated how KinA was activated and what kinds of molecular mechanisms coordinated the appearance or activity of the phosphorelay components during the course of sporogenesis upon nutrient starvation. Known as a key-player of that crucial process, the kinA gene was targeted, where it was cloned in a pDR111 vector for random mutagenesis purposes. The ultimate goal of this study was to understand how KinA recognizes environmental stimuli and transfers subsequent signals to Spo0A using B. subtilis as our model. 


\section{MATERIAL AND METHODS}

\section{Bacterial strains and plasmids}

The different strains used in this study are displayed in Table 1. Our selected vector was the pMF306 (pDR111/ $\mathrm{P}_{\text {hyper-spank }}-$-kinA-spec $^{R}$ ), which is exclusively maintained in Escherichia coli DH5 $\alpha$.

\begin{tabular}{|c|c|c|c|c|}
\hline Strain number & Organism & Genotype & Phenotype & Source \\
\hline MF1 & B. subtilis & 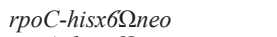 & {$\left[\mathrm{Spo}^{+} ; \mathrm{Amy}^{+} ; \mathrm{His}^{-} ; \mathrm{Neo}^{\mathrm{R}}\right]$} & Fujita et al., 2005 \\
\hline MF13 & B. subtilis & spoA::hisx $6 \Omega$ neo & {$\left[\mathrm{Spo}^{-} ; \mathrm{Amy}^{+}, \mathrm{His}^{-} ; \mathrm{Neo}^{\mathrm{R}}\right]$} & Fujita et al., 2005 \\
\hline MF60 & B. subtilis & rpoC::rpoC-gfp-spc & {$\left[\mathrm{Spo}^{+} ; \mathrm{Amy}^{+} ; \mathrm{Spc}^{\mathrm{R}} ; \mathrm{GH}\right.$} & Fujita et al, 2005. \\
\hline PY79 & B. subtilis & Wild-type & & Fujita et al., 2005 \\
\hline MF750 & B. subtilis & thrC::P-Spo ${ }_{\mathrm{IIG}}$-lacZ-erm & $\mathrm{ry}^{\mathrm{R}}$ & Fujita et al., 2005 \\
\hline MF928 & B. subtilis & $\operatorname{kin} A \because: k i n A-g f p ; s p c$ & spe & This study \\
\hline MF1237 & B. subtilis & kinA $A::$ tet & $\mathrm{et}^{\mathrm{R}}$ ] & Fujita et al., 2005 \\
\hline MF1887 & B. subtilis & $\operatorname{kin} A^{\prime} \Omega \mathrm{P}-k i n A-s p c$ & $\left.\mathrm{ny}^{+} ; \mathrm{Cm}^{\mathrm{R}}\right]$ & Fujita et al., 2005 \\
\hline MF1913 & B. subtilis & $\operatorname{kin} A^{\prime} \Omega \mathrm{P}-k i n A-c m$ & ny $;$ Thr $^{-}$Tet $^{\text {R }} ;$ Ery $\left.^{\mathrm{R}} ; \mathrm{Kan}^{\mathrm{R}}\right]$ & Fujita et al., 2005 \\
\hline MF1996 & B. subtilis & kin $A^{\prime} \Omega \mathrm{P}-k i n A-g f p ; s p c ; k a n$ & $\left.\mathrm{my}^{+} ; \mathrm{Spc}^{\mathrm{R}} ; \mathrm{Kan}^{\mathrm{R}}\right]$ & Fujita et al., 2005 \\
\hline MF2250 & B. subtilis & amyE::P-kinA-kan & $\left.n y^{-} ; \operatorname{Kan}^{\mathrm{R}}\right]$ & Fujita et al., 2005 \\
\hline MF2556 & B. subtilis & $\operatorname{kin} A \Omega \mathrm{P}-k i n A-c m ; \operatorname{th} r C: \because \mathrm{P}$ & {$\left[\mathrm{Spo}^{+} ; \mathrm{Amy}^{+} ; \mathrm{Thr}^{-} ; \mathrm{Cm}^{\mathrm{R}} ; \mathrm{Ery}^{\mathrm{R}}\right]$} & Fujita et al., 2005 \\
\hline MF2678 & B. subtilis & kinA $A::$ tet; thrC::PspoII-1 & {$\left[\right.$ Spo $;$ Amy $^{+} ;$Thr $^{-}$Tet $^{\mathrm{R}} ;$ Ery $\left.^{\mathrm{R}}\right]$} & This study \\
\hline MF2715 & B. subtilis & $\begin{array}{l}\text { amyE::P-kinA-kan;kinA } \Delta: \\
\quad \text {-lacZ;erm }\end{array}$ & {$\left[\mathrm{Spo}^{+} ; \mathrm{Amy}^{-} \mathrm{Thr}^{-} ; \mathrm{Tet}^{\mathrm{R}} ; \mathrm{Ery}^{\mathrm{R}} ; \mathrm{Kan}^{\mathrm{R}}\right]$} & This study \\
\hline MF2716 & B. subtilis & $\begin{array}{l}\text { amyE::P-kinA-kan;kinA } \\
\quad \text {-lacZ;erm }\end{array}$ & ny"; Thr"; Tet $^{\mathrm{R}} ;$ Ery $\left.^{\mathrm{R}} ; \mathrm{Kan}^{\mathrm{R}}\right]$ & This study \\
\hline $2248($ DH5 $\alpha)$ & E. coli & Wild-type & & Fujita et al., 2005 \\
\hline XL1-Red & E. coli & mutD; mutS; mutt & {$\left[\mathrm{MutD}^{-} ; \mathrm{MutS}^{-} ; \mathrm{MutT}^{-}\right]$} & Stratagene \\
\hline
\end{tabular}

\section{Media}

We used the Luria broth (LB) liquid and solid media, supplemented with appropriate antibiotics at their effective concentrations when necessary. For very specific culture needs, we also used the super optimal broth with catabolite repression (SOC) medium, the Difco sporulation medium ( $\mathrm{SM}$ ) and the nutrient broth (NB) medium for spore count purposes. We also used the $\mathrm{CH}$ medium (a rich medium in which casein hydrolysate is the sole carbon source). In addition, SpC, SpII and SpCII-EGTA media were used to prepare and store competent cells whereas starch plates proved to be helpful for the Amy test.

\section{Chemicals}

Many different chemicals were used, mostly: isopropyl $\beta$-D-1-thiogalactopyranoside (IPTG), ortho-nitrophenyl- $\beta$-galactoside (ONPG) and 5-bromo-4-chloro-3-indoyl- $\beta$-Dgalactopyranoside (X-Gal).

\section{Culture conditions}

Liquid and solid cultures were carried out under standardized conditions of temperature $\left(37^{\circ} \mathrm{C}\right)$ unless specified otherwise by the experimental procedure. For appropriate screening, the media were supplemented with antibiotics using effective concentrations. 


\section{Strain construction}

We constructed the strain MF2678 using MF1237 as recipient and MF750 as donor of DNA. In addition, we obtained the strain MF2715 by transforming the previous MF2678 using MF2250 as donor. Selection was performed on LB plates supplemented with kanamycin, erythromycin and tetracycline. We screened on starch plates in order to select only Amy (minus) colonies, which would have demonstrated Amy integration.

\section{PCR-based random mutagenesis experiments}

The primer sequences used for our in vitro amplification reaction were: OP1 (forward) 5'-GCCAAGCTTACATAAGGAGGAACTACTATGGAACAGGATACGCAGCATGTT-3', and OP2 (reverse) 5'-CGGGCATGCTTACGCCGCGAGCTGCCCTGCGAT-3'. For the DNA amplification, we proceeded as follows: $10 \mathrm{X}$ error-prone polymerase chain reaction (PCR) buffer (Invitrogen); $50 \mathrm{mM} \mathrm{MgCl} ; 0.5 \mathrm{mM}$ dATP and dGTP; $1 \mathrm{mM} \mathrm{dCTP} \mathrm{and} \mathrm{dTTP,}$ and $300 \mathrm{ng} / \mu \mathrm{L}$ of each primer. Reactions were hot-started at $95^{\circ} \mathrm{C}$ for 5 min before addition of $1.25 \mathrm{U} \mathrm{Taq}$ polymerase (Invitrogen), followed by 40 cycles $\left(30 \mathrm{~s}\right.$ at $95^{\circ} \mathrm{C}, 30 \mathrm{~s}$ at $62^{\circ} \mathrm{C}$, and 30 $\mathrm{s}$ at $72^{\circ} \mathrm{C}$ ), and a final extension step of $5 \mathrm{~min}$ at $72^{\circ} \mathrm{C}$. PCR products were analyzed using a $2 \%$ agarose gel stained with ethidium bromide and directly visualized under UV illumination.

\section{Random mutagenesis by error-prone XL1-Red E. coli}

We used XL1-Red competent E. coli cells (Stratagene). For each transformation reaction, an aliquot of $50 \mu \mathrm{L}$ XL1-Red competent cells was kept in a prechilled 1.5-mL tube. A volume of $\beta$-mercaptoethanol $(0.85 \mathrm{~mL})$ was then added to each $50 \mu \mathrm{L}$ competent cells. Tubes were swirled and then incubated on ice for $10 \mathrm{~min}$. A plasmid DNA volume of $5 \mu \mathrm{L}$ $\left(\mathrm{pMF} 306 / \mathrm{pDR} 111-\mathrm{kinA} ; \mathrm{amp}^{\mathrm{R}}\right.$ ) was added to each aliquot of cells. Tubes were incubated for $30 \mathrm{~min}$, then heat-pulsed in a $42{ }^{\circ} \mathrm{C}$ heat-block for $45 \mathrm{~s}$, and thereafter incubated for $2 \mathrm{~min}$ in ice. Finally, we added $0.9 \mathrm{~mL}$ pre-warmed (to $37^{\circ} \mathrm{C}$ ) SOC medium to each tube and incubated at $37^{\circ} \mathrm{C}$ for $1 \mathrm{~h}$ with shaking.

\section{Phenotypic screening}

Petri dishes with solid LB medium were supplemented with appropriate antibiotics at the effective concentrations. Bacteria were grown at $37^{\circ} \mathrm{C}$ overnight in an incubator. From a master plate ( $\mathrm{LB}+$ antibiotic) with well-indexed positions (dots) of each potential mutant strain and controls (MF2715 $=>\left[\mathrm{Amy}^{-}\right]$and MF60 $\Rightarrow>\left[\mathrm{Amy}^{+}\right]$), we produced replicates on Petri dishes containing solid LB medium mixed with starch, which were used to determine whether a given strain still possessed amylase activity or not. Iodine was used to spot amylase effective strains (luminescent), while amylase defective ones (null) did not glow. The phenotype of nonsporulating bacterial strain was revealed by streaking on LB medium supplemented with IPTG $(100 \mu \mathrm{L} / \mathrm{mL}$ ). Appropriate controls (positive and negative) were used. Enzymatic activity of the selected mutants was roughly evaluated previously on the LB medium containing adjusted antibiotic concentrations, IPTG $(100 \mu \mathrm{L} / \mathrm{mL})$ and X-Gal $(40 \mu \mathrm{g} / \mathrm{mL})$. Plates were incubated at $37^{\circ} \mathrm{C}$ overnight. Bacterial growth was monitored during three consecutive days. 


\section{Spore count}

We incubated a single colony in $5 \mathrm{~mL} \mathrm{LB}$ at $37^{\circ} \mathrm{C}$. At mid-log phase, an appropriate volume of the original solution was transferred to $20 \mathrm{~mL} 1 / 4$ yeast extract (YE) medium so that the resulting $\mathrm{OD}_{600}$ would be around 0.05 . The culture was thereafter shaken at $37^{\circ} \mathrm{C}$ until mid-log phase, and then $40 \mu \mathrm{L} 10 \mathrm{mM}$ IPTG was added and the cultures were incubated overnight with shaking. Afterwards, serial dilutions were made $\left(10^{-1}\right.$ to $\left.10^{-6}\right)$ for each culture. Furthermore, $100 \mu \mathrm{L} 10^{-6} \mathrm{di}-$ lution was plated on NB for viable count. Heat-kill treatment $\left(10 \mathrm{~min}\right.$ at $\left.80^{\circ} \mathrm{C}\right)$ was applied on the remaining cultures, and $100 \mu \mathrm{L}$ of appropriate dilutions was plated on NB medium for spore count.

\section{$\beta$-galactosidase assay}

For preculture, a single colony was incubated in $5 \mathrm{~mL} \mathrm{LB}$ and shaken at $37^{\circ} \mathrm{C}$. At mid-log phase, an appropriate volume of the original solution was transferred to $20 \mathrm{~mL} 1 / 4$ YE medium so that the resulting $\mathrm{OD}_{600}$ was around 0.05 . Thereafter, the culture was grown at $37^{\circ} \mathrm{C}$ with shaking to mid-log phase, and finally, $40 \mu \mathrm{L} 10 \mathrm{mM}$ IPTG was added. Every 30 min, samples were collected in duplicate accordingly. The volume collected was $100 \mu \mathrm{L}$. Cells were collected every $30 \mathrm{~min}$, spun down and frozen at $-80^{\circ} \mathrm{C}$ after $0 \mathrm{D}_{600}$ was taken. Samples were thawed at $30^{\circ} \mathrm{C}(5 \mathrm{~min})$. One milliliter Z-buffer was added and dummy reactions were created for blanks. Afterwards, $10 \mu \mathrm{L}$ lysozyme was added. At intervals, tubes were closed, vortexed and incubated for $15 \mathrm{~min}$ at $30^{\circ} \mathrm{C}$.

Thereafter, $200 \mu \mathrm{L}$ ONPG was added and the time was recorded. The mix was incubated at $30^{\circ} \mathrm{C}$ until yellow enough or for $1 \mathrm{~h}$. A volume of $500 \mu \mathrm{L}$ sodium carbonate $\left(\mathrm{Na}_{2} \mathrm{CO}_{3}\right.$, $1 \mathrm{M})$ was added to stop the reaction and the time recorded. Tubes were vortexed, and refrigerated at $4^{\circ} \mathrm{C}$ until ready for readings. Sample readings were performed according to the following procedure: samples were pre-warmed to room temperature and vortexed for $5 \mathrm{~s}$ each. Each group of 10-11 sample strain was treated together: tubes were spun for 3-10 min, and then $\mathrm{OD}_{420}$ along with $\mathrm{OD}_{550}$ was read.

\section{Nucleotide sequencing}

Amplification before sequencing was done according to the same protocol as that of PCR-based random mutagenesis. The sequencing was performed using SeqWright DNA Technology Services (Houston, TX, USA). Afterwards, using the ClustalW program, which is a general purpose multiple sequence alignment program for DNA or proteins, we obtained biologically meaningful multiple sequence alignments of divergent sequences. The ClustalW program automatically calculates the best match for the selected sequences, and lines them up so that the identities, similarities and differences can be seen.

\section{KinA-GFP transcriptional fusion constructs}

We used each KinA mutant as recipient and MF928 as donor of DNA. For GFP expression check, newly constructed KinA-GFP strains were grown on $5 \mathrm{~mL} \mathrm{LB}$, transferred to 250 -mL flasks with $40 \mathrm{~mL} 1 / 4 \mathrm{YE}$, when $\mathrm{OD}_{600} \sim 0.5$ (resulted $\mathrm{OD}_{600} \sim 0.05$ ). At OD ${ }_{600}$, IPTG was added (final concentration of $20 \mu \mathrm{M}$ ). After $30 \mathrm{~min}$ of incubation, samples of $0.5 \mathrm{~mL}$ were col- 
lected, centrifuged, pelleted, and resuspended in $50 \mu \mathrm{L}$ PBS. Readings were performed with a confocal microscope (Olympus BX 61).

\section{Triggering efficient sporulation by artificial induction of KinA synthesis}

Cells of strains harboring IPTG-inducible KinA constructs in wild-type, kinA null mutant, and spoOA null mutant were grown in CH medium and treated with IPTG at the midexponential phase of growth. Cells of wild-type strain PY79 were induced to sporulate in SM sporulation medium. Cells were treated with vital membrane stain FM4-64 at $3 \mathrm{~h}$ after resuspension in SM medium for wild-type PY79 or after treatment with and without inducer (IPTG) for the strains harboring IPTG-inducible KinA constructs, and observed by fluorescence microscopy (Olympus BX 61).

\section{RESULTS}

\section{Inducing the synthesis of KinA on solid medium}

We noticed in our preliminary results that inducing the synthesis of KinA during growth triggers efficient sporulation. In order to further explore that eventuality, we attempted to activate the phosphorelay in growing cells by artificially inducing the synthesis of KinA, which is known to feed phosphoryl groups into the relay. For that purpose, we used a fusion of kinA and the IPTG-inducible, hyper-spank promoter (hereafter referred to as $\mathrm{P}_{\text {hyper-spank }}$ ).

Results shown in Figure 1 demonstrate that the addition of IPTG to cells harboring the $\mathrm{P}_{\text {hyper-spank }}-$ kinA construct led to the appearance of many cells (about $60 \%$ of the population) that contained a polar septum, distinguishable sign that they successfully underwent sporulation.

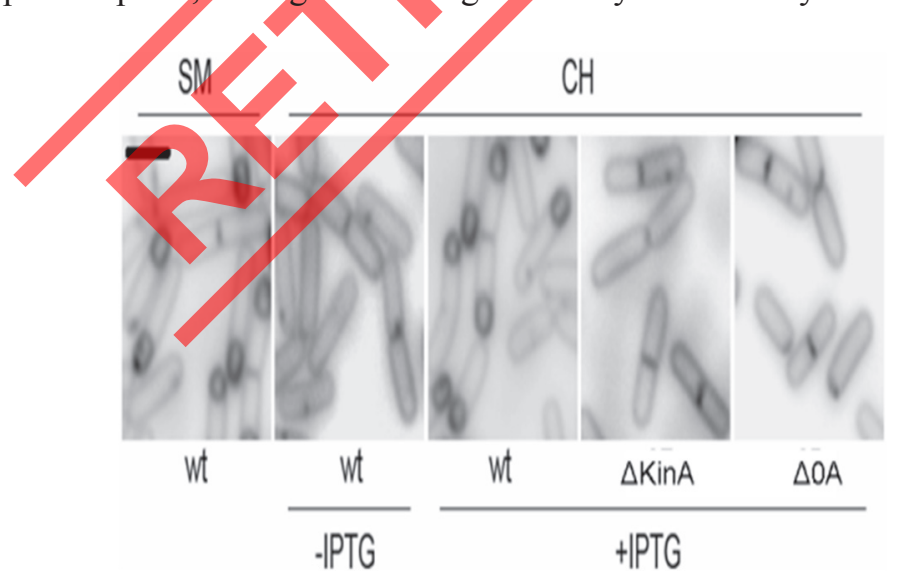

Figure 1. Triggering efficient sporulation by artificial induction of KinA synthesis. Cells of strains harboring isopropyl $\beta$-D-1-thiogalactopyranoside (IPTG)-inducible KinA constructs in wild-type (wt), kinA null mutant, and spo0A null mutant were grown in casein hydrolase $(\mathrm{CH})$ medium and treated with IPTG at the mid-exponential phase of growth. Cells of wild-type strain PY79 (wt) were induced to sporulate in sporulation medium (SM). Cells were treated with the vital membrane stain FM4-64 at hour 3 after resuspension in SM medium for wild-type PY79 (wt) or after treatment with and without inducer (IPTG) for the strains harboring IPTG-inducible KinA constructs, and observed by fluorescence microscopy. Bar $=\sim 2 \mu \mathrm{m}$. 
For comparison, only a low percentage (less than $1 \%$ ) of cells harboring the construct produced polar septa in the absence of added inducer. The percentage of cells exhibiting a polar septum was similarly small (less than $1 \%$ ) when wild-type cells (that is, cells lacking the $\mathrm{P}_{\text {hyper-spank }}$-kinA construct) were grown in $\mathrm{CH}$ medium. Furthermore, only a small number with polar septum formation was observed in the presence of inducer when cells harbored both the $\mathrm{P}_{\text {hyperspank }}-$ kinA construct and a kinA or spo0A mutation (Figure 1).

\section{Effect of inducing KinA synthesis on the cell growth}

We next demonstrated that KinA was effective in triggering rapid sporulation early in exponential phase growth. Treatment with IPTG, at an early growth stage when the cellpopulation density was low, led to a marked decrease in the growth rate after a delay of about 2 $\mathrm{h}$ (Figure 2A). We also examined this effect on solid medium. Results showed that the addition of IPTG to solid medium led to growth inhibition of cells (Figure 2B). In contrast, normal cell growth was observed when the IPTG-inducible strain also carried an spoOA mutation (Figure 2B). These phenotypes were used for the KinA mutant screening.

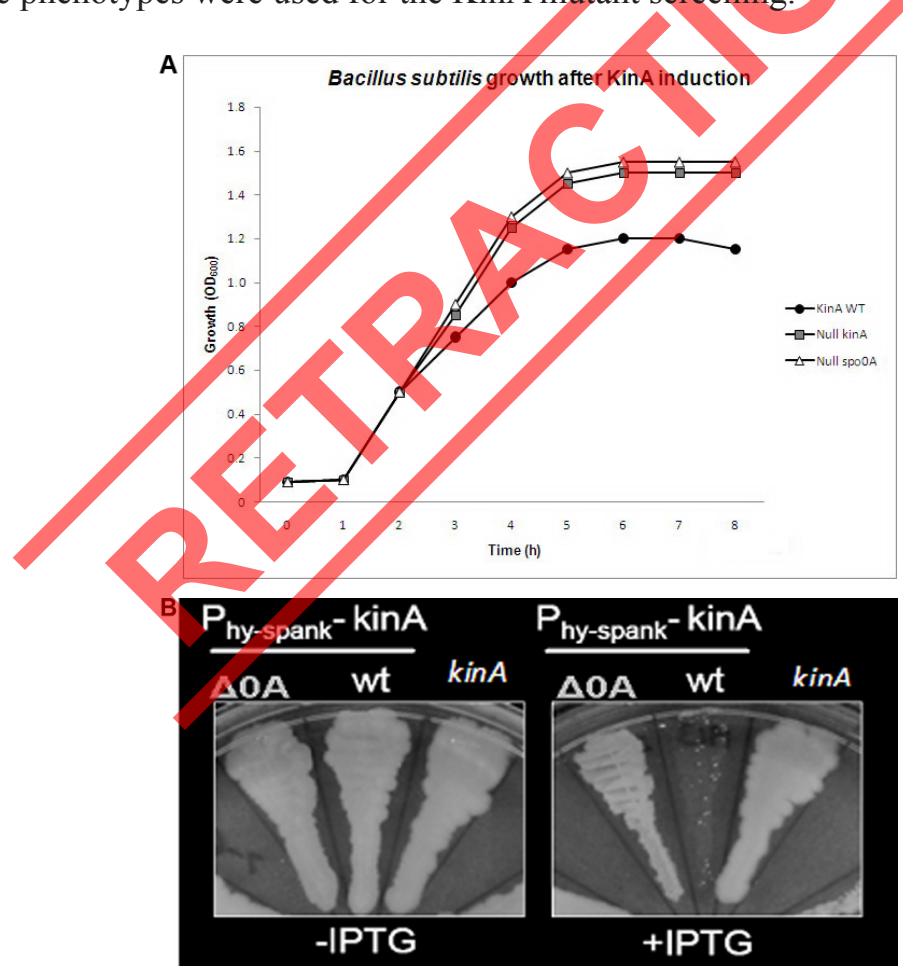

Figure 2. Effect of inducing KinA synthesis on the cell growth. A. Effect of inducing KinA synthesis on the growth rate of cells of strain harboring $\mathrm{P}_{\text {hyper-spank }}$-kinA growing in $\mathrm{CH}$ medium. Isopropyl $\beta$-D-1-thiogalactopyranoside (IPTG) was added at $\mathrm{OD}_{600}=0.05$ (circles). Null spo0A strain (triangles) and null kinA (squares) growth were also recorded. No IPTG was added as a control. Cell growth was followed by turbidity measurements $\left(\mathrm{OD}_{600}\right)$. B. Cells of wild-type (WT), null spo0A and KinA-inducible strains were streaked on the LB-agar medium in the presence and absence of $0.5 \mathrm{mM}$ IPTG, and grown overnight at $37^{\circ} \mathrm{C}$. 


\section{Isolation of growth defect kinA mutants on solid medium}

Inducing the synthesis of KinA triggered sporulation thereby inhibiting another round of DNA replication, cell division, and finally growth. While conducting the studies previously described, we noticed that small colonies arose and appeared to have survived due to impaired sporulation. We thus sought to obtain strains carrying a mutant kinA whose induction would suppress the growth defect caused by inducing KinA.

Next, spontaneous mutants (revertants) that were able to grow into colonies on medium containing IPTG were sought. Those mutants arose at a frequency of $5 \times 10^{-5}$. For confirmation purposes, independent colonies were isolated and grown on solid LB plate to check for growth defect in the presence of IPTG (Figure 3).

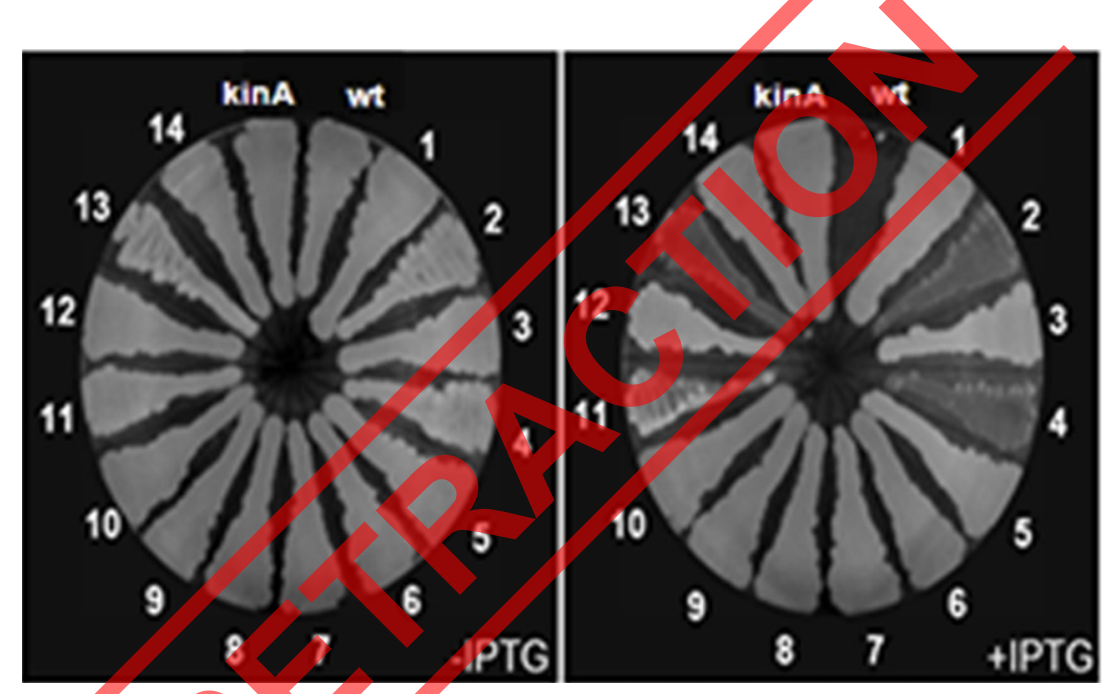

Figure 3. Phenotype associated with inducing the synthesis of wild-type and mutant kinA. Cells producing KinA in the presence of isopropyl $\beta$-D-1-thiogalactopyranoside (IPTG) were unable to form colonies (denoted $\operatorname{kinA}$ ). Growth inhibition was suppressed in the mutants (numbered). In some mutants, growth inhibition was partially suppressed (e.g., 2, 4, 11, and 13). Wild-type strain PY79 (wt, no IPTG-inducible KinA) was used as a control.

Furthermore, we hypothesized that if KinA plays a pivotal role in B. subtilis sporulation, any mutation affecting either domain would yield mutants that could not sporulate. Our method helped to improve the mutation rate by 5000 -fold, and numerous sporulation-defective mutants were generated (Figure 3).

\section{The Amy test results}

We purposely designed our system so that it could contain a kinase A gene integrated within the amylase locus (amy:: $\left.\mathrm{P}_{\text {hvper-spank }}-k i n A\right)$ and, consequently, any interesting mutant would compulsorily display a phenotype that would show a deficit in amylase synthesis [Amy-] (Figure 4). 


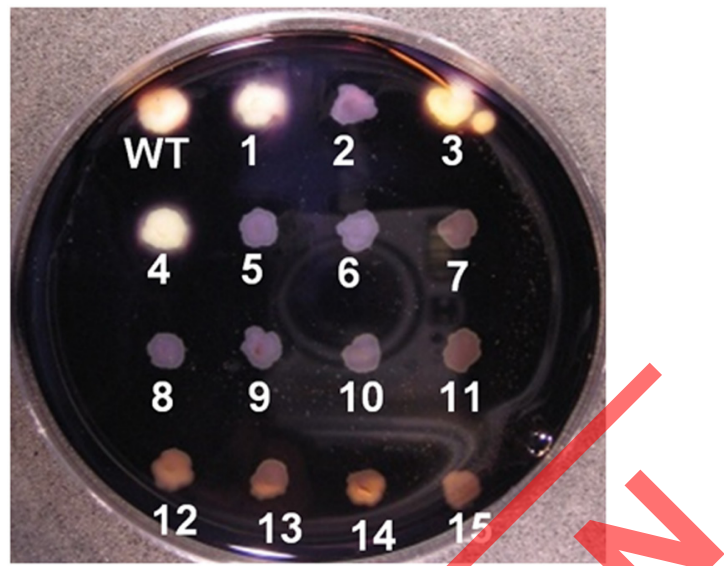

Figure 4. Screening of Bacillus subtilis Amy- mutants. The selection was conducted on LB plate supplemented with starch, natural substrate of the amylase. In case of deficiency in amylase synthesis, the colony does not glow $(2,5$ to 15$)$. When the catalytic enzyme is present, the colony glows (wild-type (WT), 1, 3 and 4).

\section{$\beta$-galactosidase activity screening on solid medium}

In order to initially investigate how the KinA recognizes environmental stimuli and transfers subsequent signals to a response regulator (Spo0A), the phenotypes displayed by the strains obtained provided a comprehensive variety of potential mutations susceptible involving some crucial KinA domains (Figure 5).

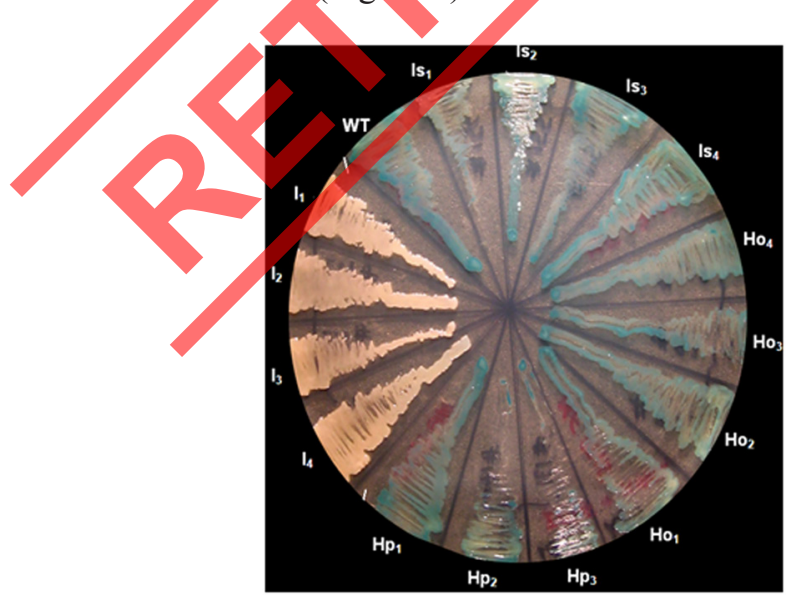

Figure 5. Bacillus subtilis sporulation-defective mutants grouped according to their phenotypes expressed on LB plate supplemented with kanamycin, tetracyclin and erythromycin in the presence of IPTG + X-GAL. Control was WT KinA [AmyE::P-hyper-spank-kinA-kan; kinA $\Delta:$ :tet; thrC::PspoII-lacZ; erm]. Observation and bacterial growth were monitored over four consecutive days. The representation of different wild-type and mutant strains growth (under IPTG) and their respective relative $\beta$-galactosidase activity during many rounds of identical random mutagenesis and screening helped in the isolation of 675 mutants belonging to 4 groups: inactive $\left(\mathrm{I}_{1}, \mathrm{I}_{2}, \mathrm{I}_{3}\right.$, and $\left.\mathrm{I}_{4}\right)$, hypoactive $\left(\mathrm{Ho}_{1}, \mathrm{Ho}_{2}\right.$ and $\left.\mathrm{Ho}_{3}\right)$, isoactive $\mathrm{Is}_{1}, \mathrm{Is}_{2}, \mathrm{Is}_{3}$, and $\left.\mathrm{Is}_{4}\right)$, and hyperactive $\left(\mathrm{Hp}_{1}, \mathrm{Hp}_{2}, \mathrm{Hp}_{3}\right.$, and $\left.\mathrm{Hp}_{4}\right)$ mutants. 
Based on the phenotypes displayed on selective Petri dishes, we were able to differentiate four groups of mutants herein referred to as inactive (no sporulation activity), hypoactive (lower sporulation efficiency than wild-type), isoactive (same sporulation efficiency as wild-type) and hyperactive (higher sporulation efficiency than wild-type), along with the wild-type strains (Figure 5).

\section{Spore count and $\beta$-galactosidase activity}

We compared the sporulation efficiency of the mutants obtained to that of the wildtype after induction (+IPTG) or without induction (-IPTG), and the results were gathered in the figure below.

For a better analysis, we then converted the sporulation percentage of the most interesting strains into a relative sporulation efficiency percentage, considering that of the wildtype as $100 \%$ (Figure $6 \mathrm{~A}$ ).

With regard to the wild-type strain taken as a reference, it appeared that we could consider the classification of the resulting mutant strains into four different categories as far as their relative sporulation efficiency was concerned.

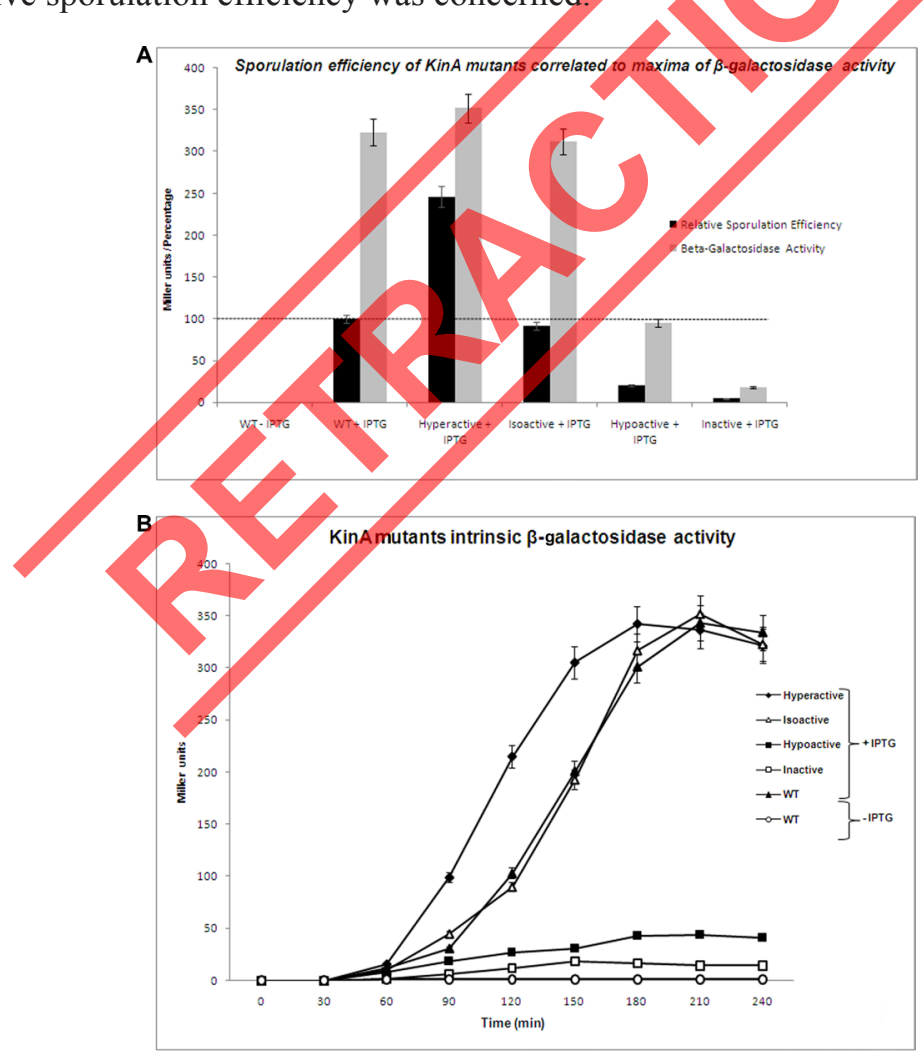

Figure 6. A. Comparative superimposition of the relative sporulation efficiency percentage and subsequent maxima of $\beta$-galactosidase activity of chemically induced Bacillus subtilis mutants in comparison to WT (+/-IPTG). B. Intrinsic $\beta$-galactosidase activities of mutants and WT ( + - IPTG) appeared to be inactive, isoactive or hyperactive. $\mathrm{WT}=$ wild-type; $\mathrm{IPTG}=$ isopropyl $\beta$-D-1-thiogalactopyranoside 
Inasmuch as in our system we designed a gene construct so that we could have the opportunity to monitor the activity of the $\beta$-galactosidase gene, which had been purposely placed under the control of the SpoIIG promoter, it became easier to test whether there was any consistency between the observed sporulation efficiency in the different described mutant strains and their intrinsic $\beta$-galactosidase activity under induction (Figure 6B).

\section{Determination of nucleotide sequences of $B$. subtilis mutants}

We expected that there would be a strong correlation between each specific phenotype and the KinA mutated sequences. Therefore, in order to further demonstrate that amino acid changes resulting from base mutations were responsible for the observed phenotypes, we determined the full nucleotide sequences of the mutants (Figure $7 \mathrm{~A}, \mathrm{~B}$ and $\mathrm{C}$ ).

As an initial approach, we targeted the inactive mutant along with the isoactive one, and the sequencing of their kinase A gene revealed a strictly conserved $\mathrm{C}$-terminus region, whereas multiple mutations caused many amino acid changes in the N-terminus region, involving more precisely the PAS-A (amino acids 5 to 116) and PAS-C domains (amino acids 267 to 333) (Figure 7A, B and C). Unlike the two other PAS domains, the nucleotide and amino acid sequences of PAS-B (amino acids 143 to 255) remained unchanged. The same observation was consistently confirmed even in hypoactive and hyperactive mutants (data not shown).

Remarkably, as we expected, all the mutants whose mutations yielded a change of the histidine 405 (autokinase active site) were unable to synthesize the functional $\beta$-galactosidase and were of course spore-defective (data not shown).

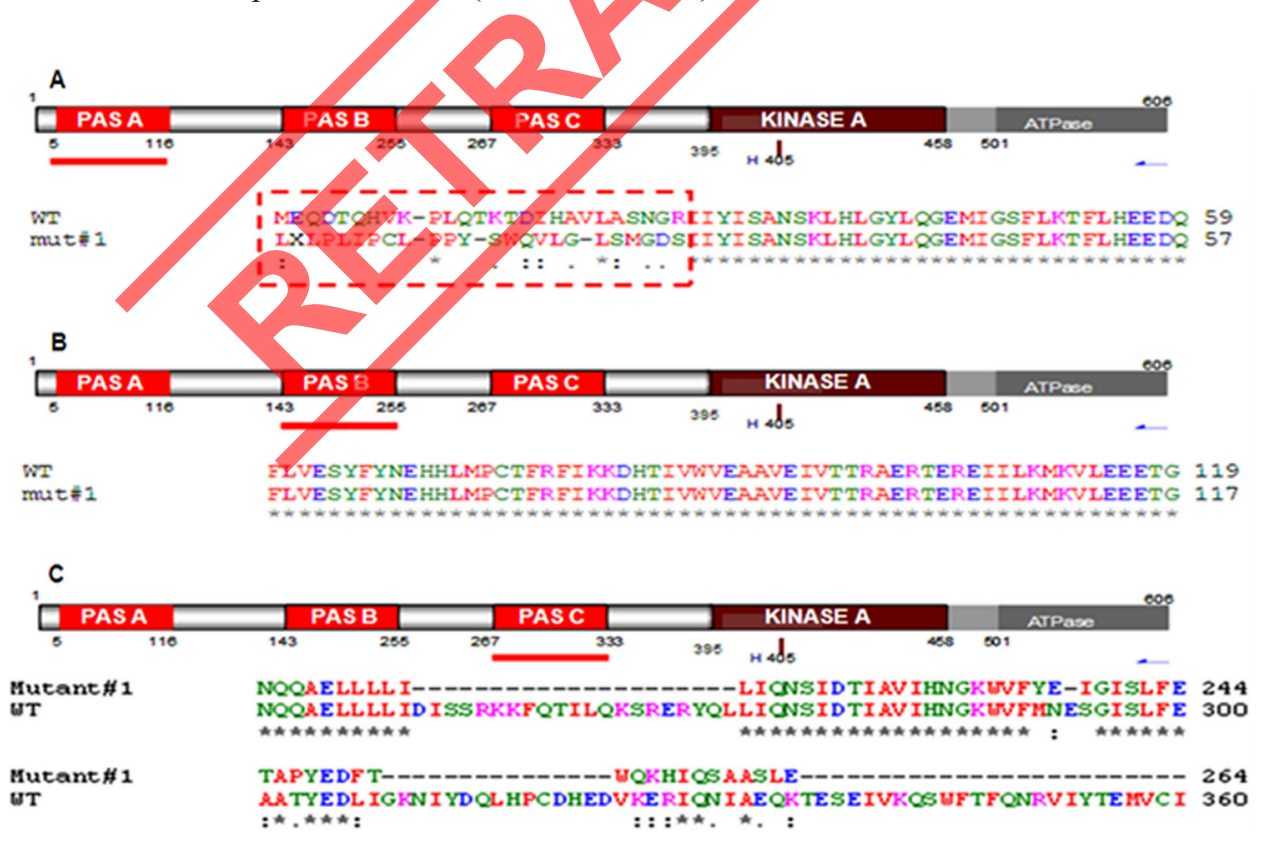

Figure 7. KinA sequence alignment of Mutant \#1 (inactive mutant) and Mutant \#2 (isoactive mutant) in comparison to wild-type (WT) using the general purpose multiple sequence alignment program for DNA or proteins, ClustalW (no gap penalty applied). Focus was exclusively on PAS domains as follows: PAS-A (A), PAS-B (B) and PAS-C (C). 


\section{KinA-GFP transcriptional fusions}

We checked whether the sporulation-defective phenotype corresponded to the synthesis of a truncated protein kinase A (nonsense mutation) or to that of a full-sized but nonfunctional protein (missense mutation).

To exclude the possibility that our spore-defective mutant originated due to the appearance of a premature stop codon within the open-reading frame, we constructed KinA-GFP transcriptional fusions, and the fluorescence level was assayed by epifluorescence confocal microscopy. Results revealed the absence of fluorescence emission in our spore-defective, inactive and hypoactive mutant cultures in comparison to that of isoactive and hyperactive mutants, along with the wild-type taken as a positive control (Figure 8).

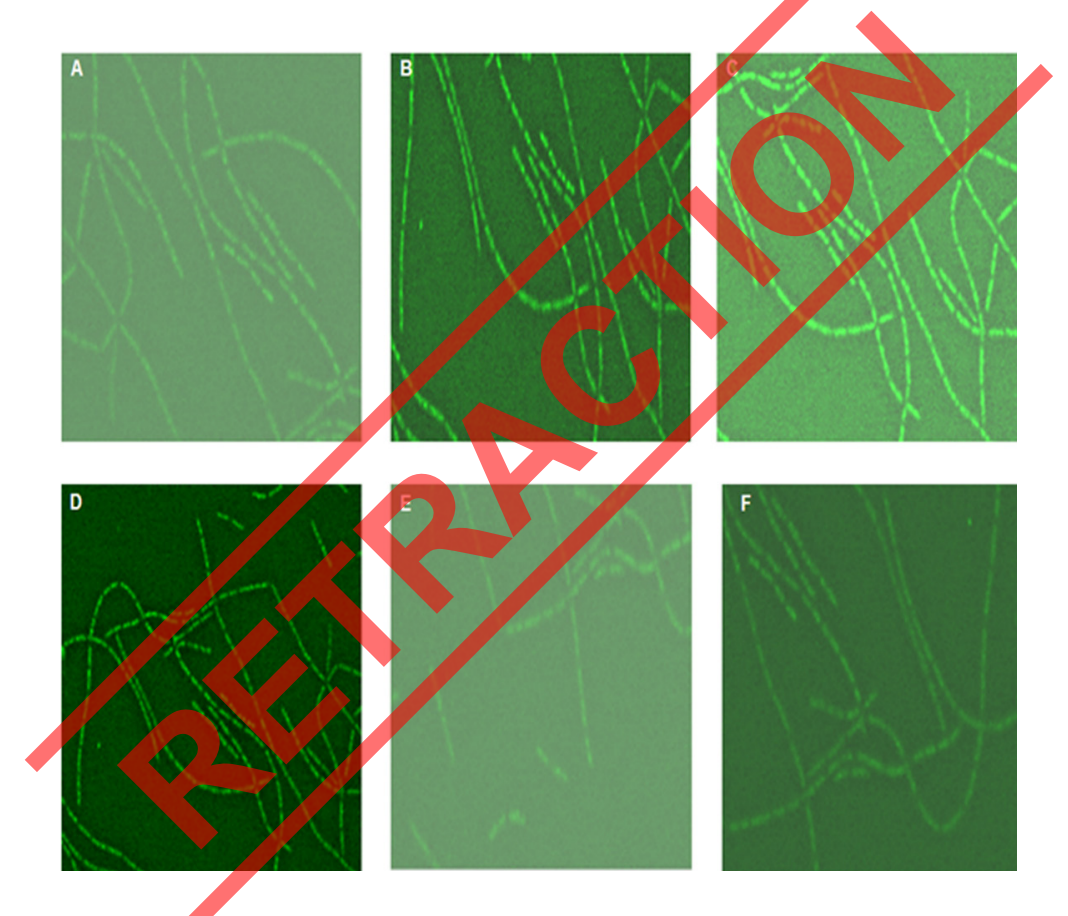

Figure 8. Green fluorescence emission of KinA-GFP transcriptional fusions. Shown on the panels are the micrographs of mutants: hypoactive (A), hyperactive (B), inactive (C), and isoactive (D); in comparison to controls WT + IPTG (E) and WT - IPTG (F). Cells were grown on $\mathrm{LB}$ at $37^{\circ} \mathrm{C}$, transferred to $1 / 4 \mathrm{YE}$ when $\mathrm{OD}_{600} \sim 0.5$, and IPTG was added (final concentration of $20 \mu \mathrm{M}$ ). After $30 \mathrm{~min}$ of incubation, samples of $0.5 \mathrm{~mL}$ were collected, centrifuged, pelleted, and resuspended in $50 \mu \mathrm{L}$ PBS and ready for observation on epifluorescence confocal microscope.

\section{DISCUSSION}

Our preliminary results indicate that entry into sporulation by inducing the synthesis of KinA is dependent on the phosphorelay. Did mutational modifications afflicted by the stringent random mutagenesis treatment efficiently affect either the active site (autokinase activity) or the signal input domain? Such alterations were chosen thanks to their utter ability to randomly mu- 
tate any single base of the kinA gene. The answer to that crucial question was partially provided by our study. First, we showed that random mutagenesis was a powerful tool to create a great variety of mutants, which enabled us to carry on a classical reverse genetics approach in order to identify KinA amino acid residues responsible for sensing environmental changes.

For such diverse panels of mutants to be obtained, mutations might have occurred throughout the whole gene structure including promoter sequences. The fact that we obtained four different types of mutants testifies in favor of the possibility to yield, using mutagenesis, novel molecules with either altered (nonsense mutation), unchanged (silent mutation) or improved (missense mutation) enzymatic activity.

Strikingly, as previously shown by Seredick and Spiegelman (2007), our results corroborated the importance of phosphorylation of Spo0A by a functional KinA, which once activated, in turn stimulates SpoIIG expression. Results showed that the addition of IPTG to solid medium led to growth inhibition of cells (Figure 2B). In contrast, normal cell growth was observed when the IPTG-inducible strain also carried an $s p o 0 A$ mutation, presumably because sporulation was impaired by blocking the phosphorelay in those cells (Figure 2B). Converging studies have shown that Spo0A is an important factor in sporulation during early mother-cell development as a mother-cell specific transcription factor (Molle et al., 2003a; Fujita et al., 2005).

Consequently, using a construct in which the $\beta$-galactosidase gene is situated downstream under the control of the SpoIIG promoter, by monitoring the $\beta$-galactosidase activity, we positively differentiated functional and non-functional KinA. From the $\beta$-galactosidase activity recorded, as expected, our results not only suggested that inactive mutants were unable to synthesize $\beta$-galactosidase but also that isoactive mutants secreted a level comparable to that of wild-type. But, unexpectedly, we did not find any substantial discrepancy (in terms of magnitude) between the wild-type and the hyperactive mutants (Figure 6B).

Moreover, we singled out that the only noticeable difference between those peculiar mutants and the wild-type resides in the early synthesis and activation of KinA in the hyperactive mutant strains, which could reasonably allow them to activate Spo0A precociously, undergo sporulation and yield more spores (Figure 6B). This result corroborates and emphasizes the importance of temporal coordination of gene expression during the sporulation process (Fujita and Losick, 2005).

It is generally believed that the phosphorelay is responsible for integrating a variety of environmental and physiological signals into the decision to sporulation (Stragier and Losick, 1996; Stephenson and Hoch, 2002).

Recent data led some researchers to conclude that Spo0A protein and activity do indeed increase in a gradual manner at the start of sporulation and that this gradual increase plays a crucial role in triggering sporulation (Fujita and Losick, 2005). An important implication of these results is that the phosphorelay is part of the mechanism for causing Spo0A and presumably Spo0A P to accumulate gradually. We, therefore, speculate that the phosphorelay plays an additional and critical role in a regulatory circuit that causes Spo0A protein and activity to increase in a gradual manner. This model was demonstrated, in part, by use of a constitutively active form of Spo0A (Spo0A-Sad, originally isolated by Grossman and colleagues) (Ireton and Grossman, 1994; Whitten et al., 2007). Thus, inducing the synthesis of the Spo0A-Sad induces acute expression of Spo0A-controlled genes and, as a result, inhibition of sporulation (Fujita and Losick, 2005).

We hypothesize that the kinetic parameter of each sporulation kinase could be one of the intrinsic determinants of a gradual increase of Spo0A activity. To assess the regulatory impact of 
a gradual activation of Spo0A, we could investigate kinetic parameters of each kinase in combination with cellular and genetic systems and biochemistry. Insights gained into the sequencing of kinA mutant genes helped to gather original data, showing that some regions appeared to be more mutation-prone than others. Thus, on the one hand, we outlined that the PAS-A (amino acids 5 to 116) and PAS-C (amino acids 267 to 333) domains belonged to that category.

On the other hand, in all mutants examined, we noticed the conservation of sequences belonging to the PAS-B and autokinase domains (Figure 7A, B and C).

Interestingly, as suggested in some parallel study, our results indicate that the PAS-A domain, notwithstanding its ATPase activity and its involvement in development (Stephenson and Hoch, 2001), still has just a minor role in KinA activity whereas PAS-B (amino acids 143 to 255 ) is proven indispensable. In addition, as previously proposed by others, our data support the evidence of the necessity of a conserved histidine residue target of the KinA during the autophosphorylation process after which the phosphate moiety is relayed via two other components of the phosphorelay to Spo0A, its final acceptor (Seredick and Spiegelman, 2007; Whitten et al., 2007).

Full appreciation of the data on the KinA-GFP transeriptional fusions showed some consistency with the previous results inasmuch as the absence of fluorescence emission in our spore-defective mutant culture in comparison to the wild-type taken as positive control corroborates the hypothesis of a truncated KinA protein (nonsense mutation). Unfortunately, we were not able to characterize a spore-defective mutant that could secrete a full-sized (but nonfunctional protein) after the occurrence of a missense mutation. We speculate that this latter event would have constituted a better candidate for our study.

Taking into account all the newly acquired data (Figure 8A-F), we managed to correlate the relative fluorescence emission with the nature of the KinA mutation borne by the bacteria. Based on those data, it appears that the missense mutations led to either hyperactive or isoactive mutants, whereas the nonsense mutations yielded hypoactive and inactive mutants. It had been demonstrated that during the initiation of sporulation, KinA was able to form a homodimer (involving PAS-B and PAS-C but notPAS-A domain) with higher concentration, which is stabilized, and resistant to protease(s) (Stephenson and Hock, 2001; Seredick and Spiegelman, 2007).

Furthermore, the same studies revealed that the PAS-A domain harbors ATPase activity, which is crucial for the sensor kinase inasmuch as it helps to translocate the phosphate to the histidine 405 at the autokinase active site. But, unfortunately, the unlikelihood of the proposed structure does not help to fully understand the enzymatic functionality of the constituted homodimer. Recent in vivo functional studies in combination with domain-based deletion analysis have shown that the cytosolic KinA forms a homodimer as an active form under both nutrient-rich and nutrient-depleted conditions via its amino- and carboxyl-terminal domains independently (Eswaramoorthy et al., 2009).

Moreover, data revealed that a mutant in which the PAS-A domain was deleted was still able to induce sporulation at the wild-type level, irrespective of nutrient availability, suggesting that PAS-BC domains are sufficient to maintain the kinase activity. They proposed that the primary role of the amino-terminal sensor domain is to form a stable complex as a functional kinase, but possibly not for the binding of an unidentified sporulation signal(s) (Eswaramoorthy et al., 2009).

Finally, when we analyze and compile all the information gathered from all the previous related studies (Hoch, 1993; Wang et al., 2001; Eswaramoorthy et al., 2009) and our present data, we are able to propose a novel hypothetical functional model of the sensor histidine- 
kinase A, which we purposely dubbed the "C-shaped intertwined model" or C-SIM (Figure 9).

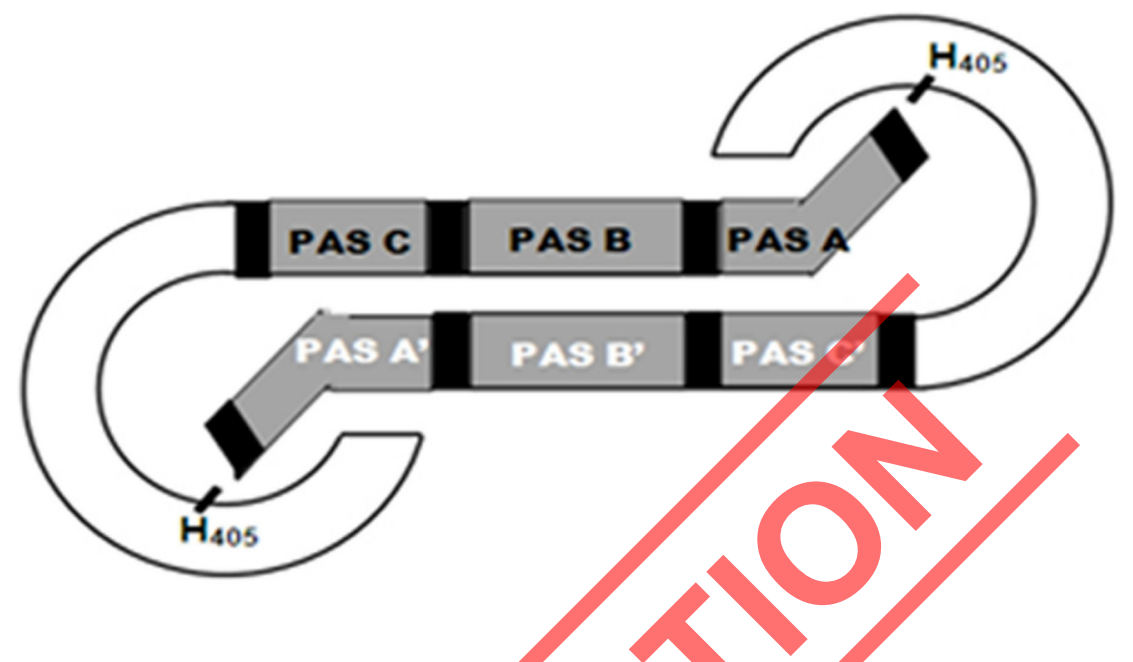

Figure 9. Schematic representation of KinA homodimerization through the hypothetical C-shaped intertwined model (C-SIM). Stable antiparallel dimeric association of two KinAmolecules (KinA/KinA'), which interact through their PAS-B domains would make it more possible for one molecule (monomere) to efficiently phosphorylate its neighbor on the histidine 405 thanks to the ATP-binding capacity of both opposite PAS-A domains.

Simply put, based on our results, we speculate that only the PAS-B and PAS-B' domains of both monomers interact strongly leading to a stable dimer and enabling the necessary interaction between the PAS-A and the autokinase domains for phosphate transfer. Meanwhile, unlike the previous models, we unyeiled that PAS-A plays a very limited role in dimerization. Accordingly, while comparing the levels of fluorescence emission of the hyperactive and isoactive mutants and that of wild-type with IPTG (positive control), there was no significant difference.

In our putative model, the structure of KinA homodimers would not only explain the gain in stability but would also exhibit more convenient architectural functional dimer units in which each monomer through its PAS-A domain, phosphorylates the neighbor's histidine 405 (spatial proximity favored by the antiparallel intertwined C-shaped structure). With respect to the C-SIM, simultaneous phosphorylations would take place on both monomers. The allosteric transitions (conformational changes) that could ensue, would allow each freshly phosphorylated PAS-A domain to be switched closer to the histidine 405 , which would, in turn, acquire the phosphate.

But, our hypothetical model, although attractive and apparently realistic, still needs to be tested. Based on our results, we propose that future aims for the continuation of this study be not only directed towards the isolation of additional extragenic mutants but also focused on the completion of their sequencing, and towards the elaboration of a site-directed mutagenesis approach, which should help to confirm the correlation between a specific mutation and its subsequent resulting phenotype. PAS domain swapping strategies and construction of a large panel of chimera kinases should also be considered.

A key challenge for the future would be to attempt to explain how the positive feedback loop leads to this gradual increase. Another fundamental challenge could be to identify which 
genes in the Spo0A regulon must be turned on or off later than others in order for differentiation to take place. Towards this aim, it could be a good idea to investigate the substrate specificity of each kinase (KinA, B, and C) in combination with cellular, genetic, and biochemical systems. Another exciting direction towards which the following steps of this study could be oriented would be the study of oxidation levels of the cell's most important substrates and coenzymes (Taylor and Zhulin, 1999). A hunt for certain specific signaling molecules such as cyclic AMP could also be interesting inasmuch as their intrinsic concentration increases when the cells are starving.

\section{ACKNOWLEDGMENTS}

Research supported by the UH-DBB under contract \#W05856-9. We also thank Tao Guo, Candice Williams, Chantal Nguyen, Esmeralda Ramirez-Peña, and Fathy Karouia for their commitment and excellent technical assistance.

\section{REFERENCES}

Becskei A, Seraphin B and Serrano L (2001). Positive feedback in eukaryotic gene networks: cell differentiation by graded to binary response conversion. EMBO J. 20: 2528-2535.

Ben-Yehuda S, Rudner DZ and Losick R (2003). RacA, a bacterial protein that anchors chromosomes to the cell poles. Science 299: 532-536.

Burbulys D, Trach KA and Hoch JA (1991). Initiation of sporulation in B. subtilis is controlled by a multicomponent phosphorelay. Cell 64: 545-552.

Dawes IW and Mandelstam J (1970). Sporulation of Bacillus subtilis in continuous culture. J. Bacteriol. 103: 529-535.

Eswaramoorthy P, Guo T and Fujita M (2009). In vivo domain-based functional analysis of the major sporulation sensor kinase, KinA, in Bacillus subtilis. J. Bacteriol. 191: 5358-5368.

Fabret C, Feher VA and Hoch JA (1999). Two-component signal transduction in Bacillus subtilis: how one organism sees its world. J. Bacteriol. 181: 1975-1983.

Ferrell JE Jr (2002). Self-perpetuating states in signal transduction: positive feedback, double-negative feedback and bistability. Curr. Opin. Cell Biol 14: 140-148.

Fujita M and Losick R (2003). The master regulator for entry into sporulation in Bacillus subtilis becomes a cell-specific transcription factor after asymmetric division. Genes Dev. 17: 1166-1174.

Fujita M and Losick R (2005). Evidence that entry into sporulation in Bacillus subtilis is governed by a gradual increase in the level and activity of the master regulator Spo0A. Genes Dev. 19: 2236-2244.

Fujita M, Gonzalez-Pastor JE and Losick R (2005). High- and low-threshold genes in the Spo0A regulon of Bacillus subtilis. J. Bacteriol. 187: 1357-1368.

Grossman AD (1995). Genetic networks controlling the initiation of sporulation and the development of genetic competence in Bacillus subtilis. Annu. Rev. Genet. 29: 477-508.

Grossman AD and Losick R (1988). Extracellular control of spore formation in Bacillus subtilis. Proc. Natl. Acad. Sci. U. S. A. 85: 4369-4373.

Hasty J, McMillen D and Collins JJ (2002). Engineered gene circuits. Nature 420: 224-230.

Hoch JA (1993). Regulation of the phosphorelay and the initiation of sporulation in Bacillus subtilis. Annu. Rev. Microbiol. 47: 441-465.

Ireton K and Grossman AD (1994). A developmental checkpoint couples the initiation of sporulation to DNA replication in Bacillus subtilis. EMBO J. 13: 1566-1573.

Isaacs FJ, Hasty J, Cantor CR and Collins JJ (2003). Prediction and measurement of an autoregulatory genetic module. Proc. Natl. Acad. Sci. U. S. A. 100: 7714-7719.

Jiang M, Grau R and Perego M (2000). Differential processing of propeptide inhibitors of Rap phosphatases in Bacillus subtilis. J. Bacteriol. 182: 303-310.

Levin PA and Losick R (1996). Transcription factor Spo0A switches the localization of the cell division protein FtsZ from a medial to a bipolar pattern in Bacillus subtilis. Genes Dev. 10: 478-488.

Mitani T, Heinze JE and Freese E (1977). Induction of sporulation in Bacillus subtilis by decoyinine or hadacidin. Biochem. Biophys. Res. Commun. 77: 1118-1125. 
Molle V, Fujita M, Jensen ST, Eichenberger P, et al. (2003a). The Spo0A regulon of Bacillus subtilis. Mol. Microbiol. 50: $1683-1701$

Molle V, Nakaura Y, Shivers RP, Yamaguchi H, et al. (2003b). Additional targets of the Bacillus subtilis global regulator CodY identified by chromatin immunoprecipitation and genome-wide transcript analysis. J. Bacteriol. 185: 1911-1922.

Perego M, Hanstein C, Welsh KM, Djavakhishvili T, et al. (1994). Multiple protein-aspartate phosphatases provide a mechanism for the integration of diverse signals in the control of development in B. subtilis. Cell 79: 1047-1055.

Piggot PJ and Losick R (2002). Sporulation Genes and Intercompartmental Regulation. In: Bacillus subtilis and its Closes Relatives: From Genes to Cells (Sonenshein AL, Hoch JA and Losick R, eds.). American Society for Microbiology, Washington D.C., 483-518.

Piggot PJ and Hilbert DW (2004). Sporulation of Bacillus subtilis. Curr: Opin Microbiol. 7. 579-586.

Predich M, Nair G and Smith I (1992). Bacillus subtilis early sporulation genes kinA, spo0F, and spo0A are transcribed by the RNA polymerase containing sigma H. J. Bacteriol. 174: 2771-2778.

Ratnayake-Lecamwasam M, Serror P, Wong KW and Sonenshein AL (2001). Bacillus subtilis CodY represses earlystationary-phase genes by sensing GTP levels. Genes Der. 15: 1093-1103.

Seredick SD and Spiegelman GB (2007). Bacillus subtilis RNA polymerase recruits the transcription factor Spo0A approximately P to stabilize a closed complex during transcription initiation. J. Mol. Biol. 366: 19-35.

Stephenson K and Hoch JA (2001). PAS-A domain of phosphorelay sensor kinase A: a catalytic ATP-binding domain involved in the initiation of development in Bacillus subtilis. Proc. Natl. Acad. Sci. U. S. A. 98: 15251-15256.

Stephenson K and Hoch JA (2002). Evolution of signalling in the sporulation phosphorelay. Mol. Microbiol. 46: 297-304.

Stragier P and Losick R (1996). Molecular genetics of sporulation in Bacillus subtilis. Annu. Rev. Genet. 30: 297-341.

Taylor BL and Zhulin IB (1999). PAS domains: internal sensors of oxygen, redox potential, and light. Microbiol. Mol. Biol. Rev. 63: 479-506.

Veening JW, Hamoen LW and Kuipers OP (2005). Phosphatases modulate the bistable sporulation gene expression pattern in Bacillus subtilis. Mol. Microbiol. 56: 1481-1494.

Wang L, Fabret C, Kanamaru K, Stephenson K, et al. (2001). Dissection of the functional and structural domains of phosphorelay histidine kinase A of Bacillus subtilis. J. Bacteriol. 183: 2795-2802.

Weir J, Predich M, Dubnau E, Nair G, et al. (1991). Regulation of spo0H, a gene coding for the Bacillus subtilis sigma H factor. J. Bacteriol. 173:521-529.

Whitten AE, Jacques DA, Hammouda B, Hanley T, et al. (2007). The structure of the KinA-Sda complex suggests an allosteric mechanism of histidine kinase inhibition. J. Mol. Biol. 368: 407-420. 